\title{
An anthropocentric approach to developing modern simulation-based decision support tools
}

\author{
C.-V. Bobeanu and F.-G. Filip \\ Research Institute for Informatics \\ 8-10 Averescu Avenue, 71316 Bucharest 1 \\ Romania \\ Phone: +40-1-2223778, Fax: +40-1-3128539 \\ e-mail: cbobeanu@roearn.ici.ro, filipf@roearn.ici.ro
}

\begin{abstract}
Rapidly changing conditions which affect manufacturing industries globally make that new demands be imposed on methods, tools and philosophies, also providing an increasing ability to companies in competing. In addition to several remarkable technology advances enabling a mimic of the human brain activity, a human centred approach is intended and pleaded for.

This paper aims at identifying those characteristic features of modern DSS that are highly demandable and acceptable by human factors, and mimic the human problem-solving. In this very perspective the authors' current results in developing a Petri nets-based simulation shell for manufacturing systems, are referred. Several interface issues are specifically dealt with.
\end{abstract}

Keywords

Decision support systems, human factors, simulation, Petri nets.

\section{INTRODUCTION}

Manufacturing industries worldwide are subject to strong paradigm shifts in the way they conceive and operate with the manufacturing systems. In this breakthrough, beside communications, extensive use of AI techniques (Kusiak 1992) is an important technology driving force. Moreover, recent concepts such as corporation reengineering (Hammer and Champy 1993) to integrate people, technologies, production and business, are offsprings of the IT advances. In turn, these developments exert a significant pressure on information systems (IS) development. In addition to technology and business efficiency factors, the human factor should be taken into account in modern IS. 
In the particular case of Decision Support Systems (DSS) Keen's (1980) remarks are of a particular relevance to this paper. He noticed that the 'final system must emerge through an adaptive process of design and usage'. The reasons why such a process include: a) the 'users concept of the task or decision situations will be shaped by the DSS, that stimulates learning and new insights, which in turn stimulates new uses and the need for new functions in the system; the unpredictability of DSS usage reflects learning, which can be exploited only if DSS evolves in response to it' and b) the 'intended users have sufficient autonomy to handle the task a variety of ways...; that suggests the user shapes the DSS'.

One special remark should be made on the new emerging concept of balanced automation (BASYS) (Camarinha-Matos and Afsarmanesh 1995) which grasps the idea of an appropriate level of technical automation, as opposed to purely anthropocentric or full automation approach. Its design and implementation need a new 're-thinking' of several areas of research, such as modelling and analysis tools and decision support tools.

This paper aims at identifying the characteristic features of more humanized modern DSS and at updating the results referred in (Bobeanu and Filip 1995b).

The material is organized as follows: Chapter 2 is concerned with the interaction and integration of human factors and information systems. Some practical results, referring the authors' work on developing DSS with characteristic features which are highly demandable and acceptable by human factors, are analysed in Chapter 3. An example comes to illustrate the described solutions, using a very flexible manufacturing system (Chapter 4).

\section{ANTHROPOCENTRIC APPROACH}

Johannsen (1994) says that 'in addition to CIM, it seems to be necessary to also consider human integrated manufacturing (HIM) during system development' and 'human centered goals have to be taken into account in addition to safety, quality and efficiency goals'.

The interaction and integration of the human and the IS have been commented in (Filip 1995). The perspective includes three main groups of questions: a) how does the computer serve the human to perform his task better? b) what is the impact of the man-machine system on the operational performance of the managed process/plant? and c) how the human status and working conditions are affected by the presence of computer?

Asking the first question, we reach the conclusion that while most of the critical issues of an early IS (i.e. unreliable, intolerant, and not self sufficient) have so far been solved by technological developments and intensive training of users, rendering the information tool less impersonal is still an open problem, at least in process supervision and control.

To answer the second question, Johannsen's remarks (Johannsen 1994) according to which the tendency to continue automating in a technology-driven manner led to 'deskilling the human operators, and thus to boredom under normal operational conditions as well as to human errors in emergency situations', are duely considered. Even though with Johannsen physical process control counts, it is human errors that severely bear on physical safety and economically at the higher decision levels such as production control and plant management.

Regarding the third question, special attention was paid to Briefs' comments (Briefs 1981) that the computerisation of human work seemed to imply a ' threat against the human creativity and conscious development, since there is a tendency to polarise human into categories': 
a)'computer professionals who manifest and develop their skills and creativity in designing ever more sophisticated tools' and b) 'communities of computer users who fast and easily perform their jobs without getting deep insights into their comfortable production means'.

To sum up these ideas, the authors' belief is that it is necessary to develop modern DSSs that are not only human centered (anthropocentric), in that they serve the human to perform his (designing/planning) task better by using the facilities that are familiar to and accepted by human factors (attractive, user-friendly interfaces, interactively controlled runs, etc), but also anthropomorphic, (in that they mimic the human problem solving) by synergising KBS and DSS, and, finally, they are not a threat against the human factor as to replacing him.

Some practical results are discussed in the sequel, referring the authors' current activity aimed at developing a Petri nets-based simulation shell for discrete production systems, and trying to outline how it can meet the above-mentioned requirements.

\section{MODCPN - A PETRI NETS-BASED SIMULATION SHELL FOR MANUFACTURING SYSTEMS}

Petri nets are an often used graphical and mathematical modelling tool for the specification and analysis of systems which can be defined as concurrent, asynchronous, distributed, parallel, non-deterministic and/or stochastic (Murata 1989). Since Petri nets actually stand for a language for discrete event systems behaviour description, they need a set of tools for assisting the user. As a survey of this field indicates, a lot of editors, simulators and analysers of the models described by such a formalism are proposed. However, most of these Petri nets tools are stand-alone tools and are not self sufficient tools.

ModCPN reflects the authors' contribution to this field. It is meant as a modelling support for both product upgrades and training and consulting. It is an open system, viewed as an integrated set of tools, managed by a supervisor and supporting the build-up and use of Petri nets-based models. The above-mentioned shell provides tools to support the user in performing different stages of the simulation process: a) modelling; b) validation-by-analysis of the dynamic and static characteristics; c) simulation and output analysis.

It can be seen as a DSS for discrete manufacturing, purposely designed and developed to meet several requirements of modern, more humanized DSS. In order to stress the utilization of skilled human resources and the participation in the decision-making, one special note should be made on the following aspects:

1.The underlying conception of the supervisor is to stimulate human decision-maker's reasoning and intuition and to promote users' conception and expertise all their activity through. In this respect, it is entirely up to the user, who has been given several 'pull-down' menus, to put together the shell components, to opt for a strategy for validation-by-analysis, and for simulation modes, to determine the conditions under which the component tools are to be used, and to establish the global parameters of the model.

2. The previous observation sets the stage for another characteristic feature of the system: its being designed for supporting and stimulating user's learning and new insights.

3. Special attention was paid to self sufficiency and unpredictability in the shell usage. A user-friendly special feature of the Windows interface and the help system structured at three levels will guide the human decision-maker in making the right choice as to the analysis 
strategy, provide best alternatives and check on his actions' consistence and suitability to the results obtained, and assist the user in iterating simulation experiments.

4. Finally, less impersonal characteristics of the information system are enabled by an intensive use of the suggestive power of the modelling tool - Petri nets - which helps to solve the problem of searching for an appropriate means of communication between system developers and system users.

A detailed description of the tools is given in (Bobeanu and Filip 1995a). It runs on IBM PC compatible computers and has been developed by applying a new object-oriented paradigm, using $\mathrm{C}++$ programming language. The envisaged Petri nets as theoretical foundation fall under the following classes: P/T nets, coloured Petri nets and Timed Transition Petri nets.

\subsection{Modelling component (MODNET)}

Thanks to the design of the graphical editor, net drawing is as natural as can be, and the user smoothly commutes from paper drawing to computer-aided drawing. Within this module different icons (places, transitions, arcs) are selected and put in a graphical modelling layout, so that the model is built up graphically (Figure 2). Additional buttons are provided to support: a)a delete function on the already drawn graphical objects; b) an insert text function and c) a selection mechanism for further details set up. As now operating in the system, the modelling function claims for a user of the model description tool having basic knowledge in the field.

The modelling function takes two clear-cut steps: a) topological (statical) description of Petri nets; b) description of the initial marking, of the colour and transition temporisation functions.

The graphical environment used for model description covers the following: a) a menu bar of which options specify main function groups of the component (file/ window/ option/ erase/ help) and a lower level menu assigned to each main option; b) a toolbox with the basic components of Petri nets; c) a central network drawing window. It will allow: a) network loading; b) network recovery; c) quit; d) actual drawing functions; e) handling data on network components and associated colour functions and temporisations; f) network components' associated information display; g) a syntactical proof of the overall network description, of the initial marking, of the colour and temporisation functions; h)working on the whole network.

\subsection{Validation-by-analysis component (ANET)}

ANET component makes it possible that the model characteristics are analysed and, provided that model inadequacies exist, that useful information on model re-definition is furnished. For example, the error-yielding structural elements of the model are to be detected (including locations with infinite accumulations of tokens, transitions which are performed on no reached marking, etc.). It is a set of method-oriented analysis tools (reachable marking tree and reduction rules) and that means classes of properties under study. Only certain properties of Petri nets (boundedness, liveness, home properties, place/transition invariants) are considered.

As low efficiency in analysis techniques is parallel to rendering the Petri nets class concerned more general, a refinement process of the analysis has been aimed at by providing unsophisticated variants. The envisaged solutions (Bobeanu 1994), given the analysis complexity, take most advantage of the impact made by network classes on general network. No established trajectory of the analysis process being possible, a man-machine dialogue will 
effectively support the process, which will be largely tributary to the features of the Petri net concerned, to the study goals, and to preliminary results. The supervisor helps the user adopt an analysis technique, by means of a 'help' system (for instance, it 'suggests', if the case is, that the reachable marking tree or the reduction rule is to be considered for studying the dynamic properties of a given Petri net). The supervisor also checks the user's solutions consistency (it will prevent the user, let's say, from taking the variant of reachable marking tree based on some structural elements, before testing via reduction rules the static properties).

\subsection{Simulation component (PENTSIM)}

The system behaviour is analysed by simulation and, if necessary, iteratively adapted to the system requirements. Two simulation strategies are available:

1. interactively controlled (user driven) simulation, which enables the user of a simulation tool to select a subset of the enabled transitions to fire. User is advised on this model appropriateness for simulation models where only some sequences of activities are interesting, or where certain single activities or single exceptional situations are to be validated. The simulation steps are stored in a system evolution file, hence it is possible to rollback to an earlier simulation state and to examine alternative simulation runs.

2. automatic simulation, based on a certain strategy: a) fire for each reached marking a maximum subset of enabled transitions; b) introduce a priority arrangement of the transitions and fire the transitions in each marking according to this ordering, such that there should be no conflict between the selected transitions.

This mechanism is based on an incrementally refined model structure, so the user faces a structure which resembles the role and nature of real-life system components. A two-subclass partition of the place set is considered: 1) places of type $A$, modelling the temporal entities advance in the model and 2) places of type $B$, for the synchronization conditions. A partition of the transition set is also made: 1) transitions of type $a$, which model normal processing phases; 2 ) transitions of type $b$, associated with places of type $B$ and 3) transitions of type $c$, which generate new temporal entities of the model.

A two-stage selection strategy is available via 'pull-down' menus for defining a temporal entities priority arrangement. So 1) associated priorities and 2) queueing strategies should be considered during the simulation, according to some given criteria: a) queue arrival time (FIFO, LIFO); b) system arrival time (First in System, Last in System) and c) last operation completion time (Earlier Steps First, Later Steps First). Thanks to the use of queueing mechanism (associated with places of type A) and of priorities (associatted with places of type A and/ or transitions) as parameters, this DSS can be seen as a balanced system.

System features and the suggestive power of coloured Petri nets help the user have a 'seize' and good grasp capacity of the simulation process subtleties.

Output analysis under Microsoft Excel will help the user evaluate performance. It will provide information about: a) min/average/max value of cycle time and processing time; b)average/valid value of work-in-progress; c) number of jobs completed for every part type.

A machine utilisation analysis (loaded/vacant/breakdown average time) is also included. The 3D graphical representation facilities, whenever applied, will make various simulation scenarios outputs very eloquent. In order to present the output data most clearly, three chart types will be offered to the user to choose among: a) a 3D bar chart type, which emphasizes the values of 
individual entity types and draws comparisons between entities (Figures 3 and 4); b) 3D perspective column chart, which allows data concerning resources utilisation are compared, and views the data by category (Figure 7); c) 3D surface chart type which is useful for finding the best combination of parameters improving the performance measure (Figures 5,6 and 7).

An example comes to illustrate the above-mentioned solutions.

\section{EXAMPLE}

On considering a very flexible manufacturing system (Figure 1), it is possible to identify four workstations $\{\mathrm{WS} 1, \mathrm{WS} 2, \mathrm{WS} 3, \mathrm{WS} 4$ \}, each one with a buffer connected to a circular conveyor. An input/output buffer is also included in the system. Three part types $\{$ WP1, WP2, WP3 $\}$ are produced in this FMS. Their processing sequence can presumably be performed on two alternative workstations and a part may have more successive operations on the same machine of the workstation WS1 or WS3. Starting with an empty line and arrivals of 2 workpieces of type WP1, 20 workpieces of type WP2 and 8 workpieces of type WP3, are taken for granted in our example.

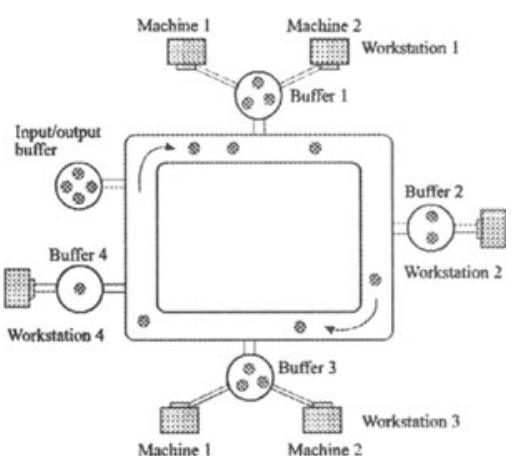

Figure 1 Overview of the FMS.

The module MODNET allows to create a Petri nets-based model description by selecting icons and by putting them in a graphical modelling layout, as shown in Figure 2 . The refined structure of the net which simulation operates with, includes the following main elements: 1)places of type $A$ : P3, P5 (buffer stations used before each production step); P8, P11 (decision nodes); 2) places of type $B$ : P4, P6 (available resources); P7 (breakdown resources); $\mathrm{P} 1, \mathrm{P} 2$ (inputs generation conditions); P9, P10 (routing conditions); 3) transitions of type $a$ : T2, T3, T4, T5 (production steps); 4) transitions of type $b$ : T6 (machine breakdown); T7 (machine set-up); T8, T9 (routing steps); T10 (inputs inhibition step); 5) transitions of type $c$ : $\mathrm{T} 1$ (input generation) with input inhibitory arc. The associated initial marking is the following: $\mathrm{M} 0(\mathrm{P} 1)=\{<1, \mathrm{IG}>\} ; \mathrm{M} 0(\mathrm{P} 4)=\{<1, \mathrm{WS} 2\rangle,<1, \mathrm{WS} 4>\} ; \mathrm{M} 0(\mathrm{P} 6)=\{<2, \mathrm{WS} 1>, \quad<2, \mathrm{WS} 3\rangle\}$; $\mathrm{M} 0(\mathrm{P} 9)=\{<1, \mathrm{SSO}>\} ; \mathrm{M} 0(\mathrm{P} 10)=\{<1, \mathrm{CC}>\}$ and all its remaining definitions indicate a null value. The set of colours associated with places/transitions is given in Table 1 and Table 2 . Many simulation scenarios have been produced through PENTSIM component for different parameters (priorities, queueing strategies) settings over a time horizon of eight hours. Figure 3 through Figure 7 show several eloquent representations of typical results of these tests.

\section{CONCLUSIONS}

An anthropocentric and anthropomorphic approach in DSS development was pleaded for in this paper, referring the authors' current activity on developing a Petri nets-based simulation shell for discrete production systems. The ModCPN simulation shell evolution will be in relation with the reassessment of the impersonal/ learning stimulating ratio by using a model base (including both predefined subnets, to be used without knowing anything about Petri nets, 
Table 1 (Places description):

\begin{tabular}{|c|c|c|}
\hline Place & Signification & Colour function \\
\hline P1 & Open inputs & $\{\mathrm{IG}\}^{*}$ \\
\hline P2 & Closed inputs & $\{I G\}$ \\
\hline P3 & $\begin{array}{l}\text { Waiting in the buffer station (full operational } \\
\text { workstation) }\end{array}$ & $\begin{array}{l}\{\text { WP12, WP14, WP22, WP24. WP32, } \\
\text { WP34\} }\end{array}$ \\
\hline P4 & Available resource (full operational workstation) & $\{$ WS2, WS4 $\}$ \\
\hline P5 & Waiting in the buffer station (part operat. workstation) & $\begin{array}{l}\text { \{WP11,WP13,WP21, WP23, WP31, } \\
\text { WP33\} }\end{array}$ \\
\hline P6 & Available resources & $\{\mathrm{WS} 1, \mathrm{WS} 3\}$ \\
\hline P7 & Breakdown resources & $\{$ WS1. WS 3$\}$ \\
\hline P8 & Decision node (subseq. op.) & $\begin{array}{l}\text { \{WP11, WP13, WP21, WP23, WP31, } \\
\text { WP33\} }\end{array}$ \\
\hline P9 & Successive operations & $\{\mathrm{SSO}\}^{21}$ \\
\hline P10 & Complete cycle & $\{\mathrm{CC}\}^{3)}$ \\
\hline P11 & Decision node (new cycle) & $\{\mathrm{WP} 1, \mathrm{WP} 2, \mathrm{WP} 3\}$ \\
\hline
\end{tabular}

Table 2 (Transitions description):

\begin{tabular}{lll}
\hline Transition & Signification & Colour function \\
\hline T1 & Input generation & $\{$ WP1. WP2. WP3\} \\
T2 & Full-time operation & $\{$ WS2, WS4\} \\
T3 & Next operation & $\{$ WS1,WS3 \\
T4 & Routing tests & $\{$ WP11, WP13, WP21, WP23, WP31, WP33, $\}$ \\
T5 & Parts outputs & $\{$ WP1. WP2, WP3\} \\
T6 & Breakdown & $\{$ WS1, WS3\} \\
T7 & Setup & $\{$ WS1, WS3 \\
T8 & Successive operations & $\{$ WS1. WS3\} \\
T9 & New cycle initiation & $\{$ WP1. WP2, WP3 \\
T10 & Inputs inhibition & $\{$ IG \\
\hline
\end{tabular}

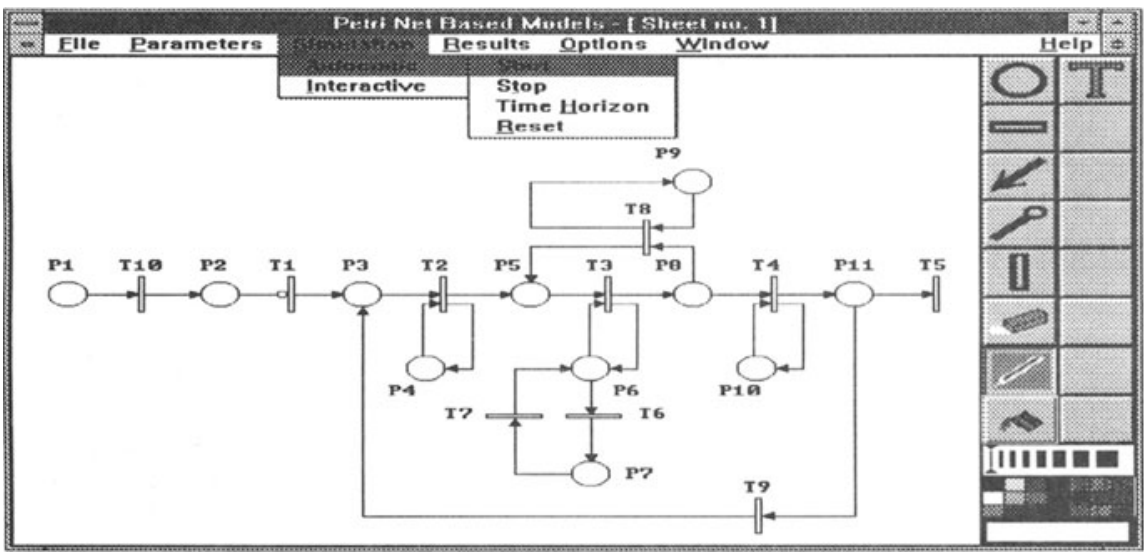

Figure 2 Petri Nets-Based Model Display with Automatic Simulation Run Menu.

\footnotetext{
") IG stands for 'Input generation'

" WPij stands for "WPi is to be processed on station WSj'

${ }^{2)}$ SSO stands for 'Successive operations'

${ }^{3)} \mathrm{CC}$ stands for 'complete cycle'
} 


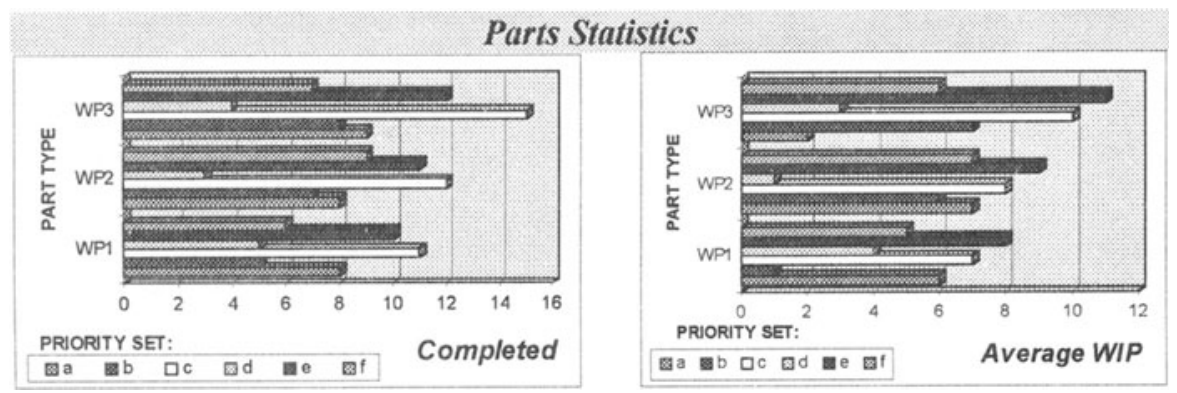

Figure 3 Number of Completed Parts per Part Type, 3D-Bar Chart Type.

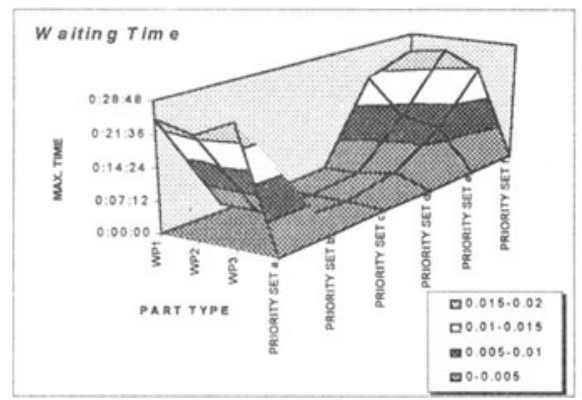

Figure 5 Maximum Waiting Time per Part Type, 3D-Surface Chart Type
Figure 4 Number of Average WIP per Part Type, 3D-Bar Chart Type.

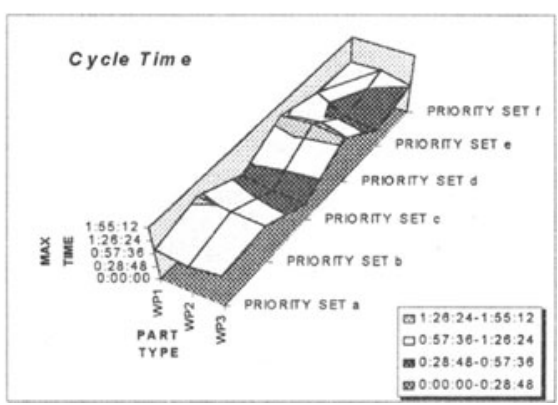

Figure 6 Maximum Cycle Time per Part Type, 3D-Surface Chart Type.

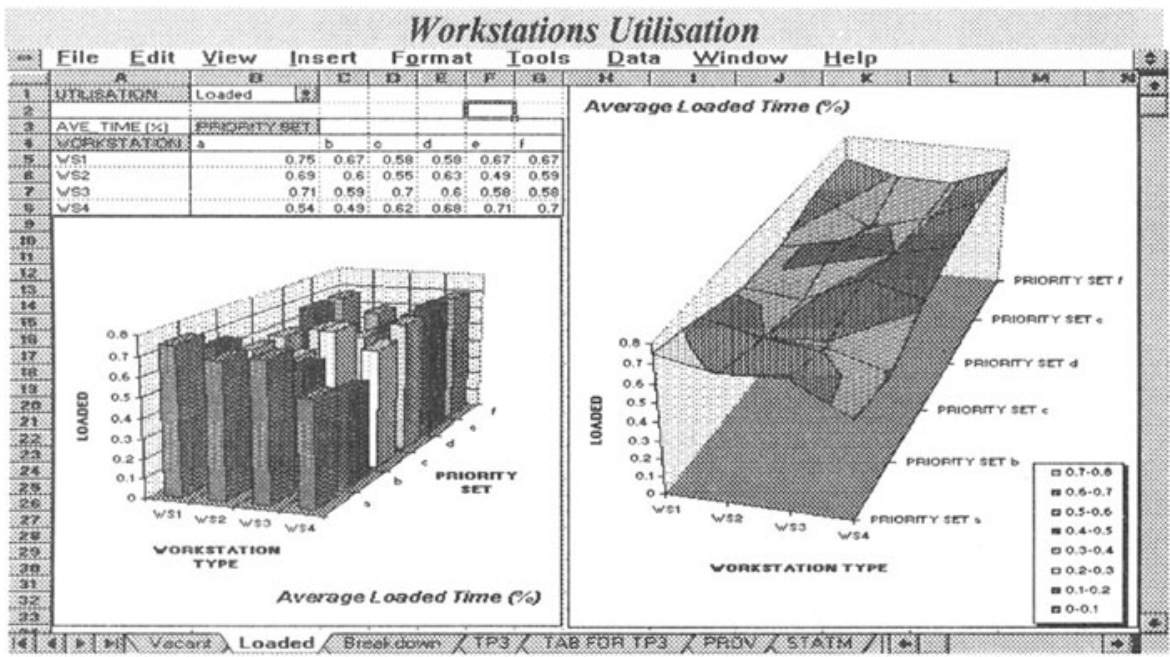

Figure 7 Average Loaded Time (\%) per Workstation, 3D-Bar and 3D-Surface Chart Types. 
and user defined subnets), which will enable the user to decide whether he really wants to become familiar with the basis of Petri nets formalism required for model editing.

\section{REFERENCES}

Bobeanu, C. (1994) On the Analysis of Certain Classes of Petri Nets. In Proceedings of the IASTED International Conference Modelling and Simulation MS' 94, Pittsburgh, PA, USA, May 2-4, 87-90.

Bobeanu, C. and.Filip, F.G. (1995a) ModCPN-An Integrated Set of Tools for Petri NetsBased Modelling of Manufacturing Systems. In Proceedings of the IEEE INRIA Conference on "Emerging Technologies and Factory Automation", Paris, October 10-13, 95-103.

Bobeanu, C. and Filip, F.G. (1995b) A Simulation Shell for More Humanized Decision Support Systems. In: Proceedings of the 7th European Simulation Symposium, ESS'95, Erlangen-Nuremberg, Germany, October 26-28, 268-272.

Briefs. V. (1981) Re-thinking industrial work: computer effects on technical white collar workers. Computers in Industry, 2 (1), 76-89.

Camarinha - Matos, L.M. and Afsarmanesh, H. (Eds.) (1995) Balanced Automation Systems. Architectures and Design Methods. Proceedings of the IEEE/ECLA/IFIP International Conference, Vitória, Brazil, 24-26 July.

Filip, F.G. (1995) Towards more humanized real-time DSS. In Balanced Automation Systems. Architectures and Design Methods. Proceedings of the IEEE ECLA IFIP International Conference, Vitória, Brazil, 24-26 July, 230-237.

Hammer M. and Champy, J. (1993) Reengineering Corporation. Harper Collins Books, New York.

Johannsen, G. (1994) Integrated Systems Engineering: The Challenging Cross-Discipline. In Preprints on "Integrated Systems Engineering" of the IFAC. Conference, Pergamon Press.

Keen, P.G.W. (1980) Adaptive Design for DSS, Data Base 11 (4), 15-24.

Kusiak, A. (Ed.) (1992) Intelligent Design and Mamufacturing. John Wiley \& Sons, New York.

Murata, T. (1989) Petri Nets: Properties, Analysis and Applications. Reprinted from Proceedings of the IEEE, 4 (77).

Carmen-Veronica Bobeanu graduated in Mathematics from the University of Bucharest in 1979. Currently she is enrolled in a $\mathrm{Ph}$. D part-time programme in Mathematics at the University of Bucharest. She is a Senior Researcher at the Research Institute for Informatics and an Associate Professor at the University of Bucharest. Her major scientific interest goes to modelling discrete event systems with emphasis on production systems, scheduling problem, Petri nets, intelligent simulation environments.

Florin-Gheorghe Filip took the M. Sc. degree and Ph.D degree from the Polytechnical Institute of Bucharest in 1970 and 1982, respectively. In 1991 he was elected as a corresponding member of the Romanian Academy. He is director of the Research Institute for Informatics in Bucharest. His main scientific interests are in large scale systems, optimisation and control, DSS, CIME, man-machine systems, technologies for the business processes. He published over 120 technical papers. 